\title{
Fatigue Analysis of Mobile Maintenance Platform Based on Ansys Workbench
}

\author{
Yongliang Yuan \\ School of mechanical engineering, Dalian University of Technology, Dalian, China
}

\begin{abstract}
In order to investigate the performance of the mobile maintenance platform, Ansys Workbench was used to analyze the strength analysis of the mobile maintenance platform. The deformation, stress, and strain were obtained. The fatigue module was used to analyze the fatigue of the mobile maintenance platform and the fatigue life based on the cumulative fatigue damage theory. The simulation results show that the strength of the mobile maintenance platform is sufficient, and its lifetime is as high as 19.8 years. The mobile maintenance platform has a large space for optimization and this paper provides a basis for future structural optimization design.
\end{abstract}

\section{Introduction}

The mobile maintenance platform is a common aerial maintenance operation device, which is mainly used to perform maintenance on the facilities in the air, such as maintenance of street lights and space decoration. Many scholars have done a lot of research on mobile platforms and achieved many research results ${ }^{[1-4]}$, which mainly focused on the kinematics and force analysis of mobile platforms. Due to different working spaces and height, mobile maintenance platform devices need constant optimization and improvement.

With the development and wide application of finite element analysis technology, it has been successfully applied in the automotive, mechanical, aerospace and other fields. In this paper, the deformation, stress, strain, and fatigue life of the mobile maintenance platform have been obtained based on an improved mobile service platform. This paper provides a basis for future structural improvement and optimization. The flowchart of the mobile maintenance platform is shown in Figure 1.

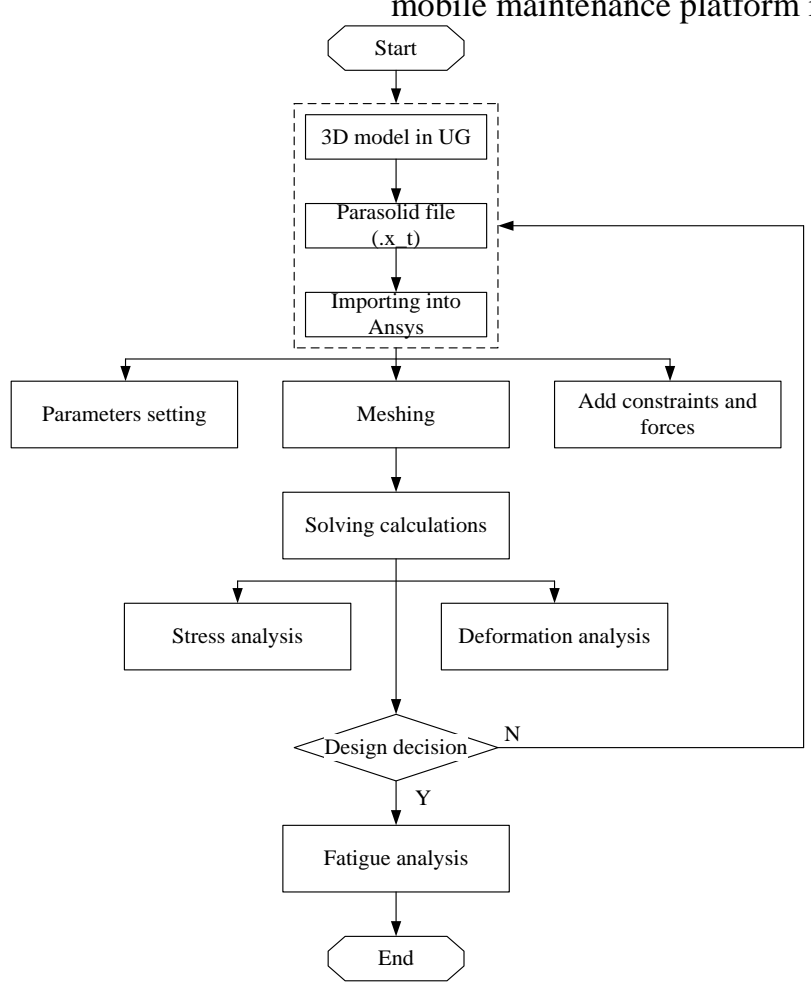

Figure 1. The flowchart of mobile maintenance platform. 


\section{The establishment of the model}

The mobile maintenance platform is an infrastructure used for the maintenance of the space equipment. Therefore, the height should not be too high, otherwise, it will easily lead to instability. There are many methods for establishing finite element model, which can be mainly divided into finite element modeling and CAD modeling. This paper uses UG software to establish the 3D model. The width of the mobile maintenance platform is $2 \mathrm{~m}$ and the height is $5 \mathrm{~m}$. The model is imported into the Ansys/Workbench. The structure of the model is shown in Figure 2.

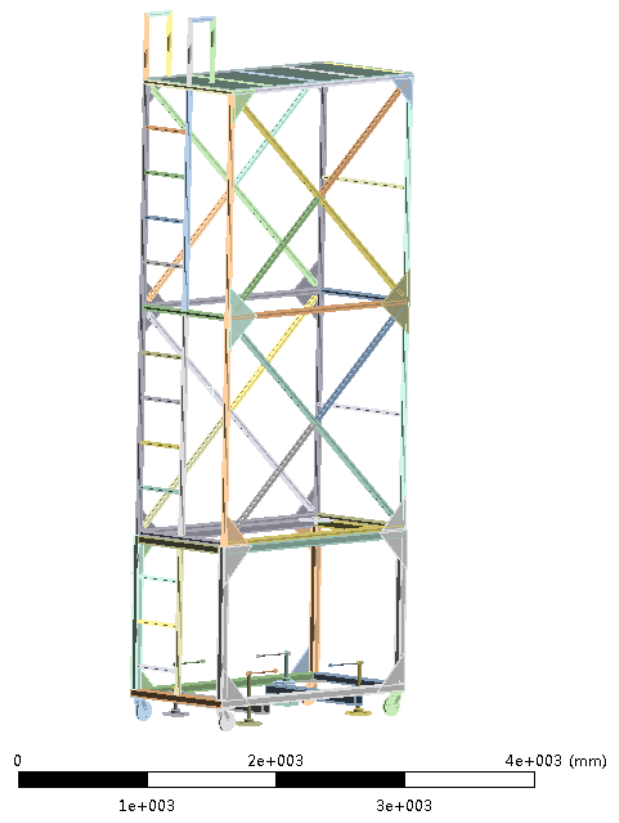

Figure 2. Mobile maintenance platform.

\section{Static analysis}

The finite element model usually includes a geometric model, material properties, and meshing. Static analysis includes applying loads and constraining boundary conditions and solving.

\subsection{Definition of material properties}

The wheels made by LC4 aluminum alloy, the main mechanical properties of this material include: elastic modulus is $66.64 \mathrm{GPa}$; Poisson's ratio is 0.31 ; density is $2850 \mathrm{~kg} / \mathrm{m}^{3}$, and strength limit is $550 \mathrm{MPa}$. The remaining shelves and other materials for the mobile maintenance platform are selected from structural steel, which is the default material in Ansys/Workbench.

\subsection{Meshing}

In order to get an accurate finite element model, this paper selects the unit size is $50 \mathrm{~mm}$. The grid of mobile maintenance platform is shown in Figure 3, including 81,656 units and 255,875 nodes.

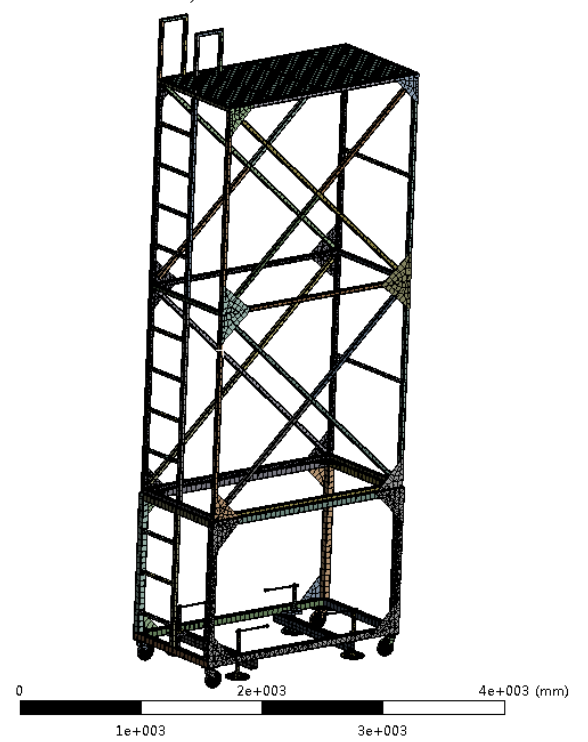

Figure 3. The grid of mobile maintenance platform.

\subsection{Software implementation}

Assume that the mobile platform needs to be able to withstand three people working on it at the same time. Therefore, the weight is selected as a constraint, and the plane of the bases is used as a constraint, in which the gravity of the mobile platform is considered. After adding the loads and constraints, the finite element static analysis of the model is solved. The stress is shown in Figure 4.

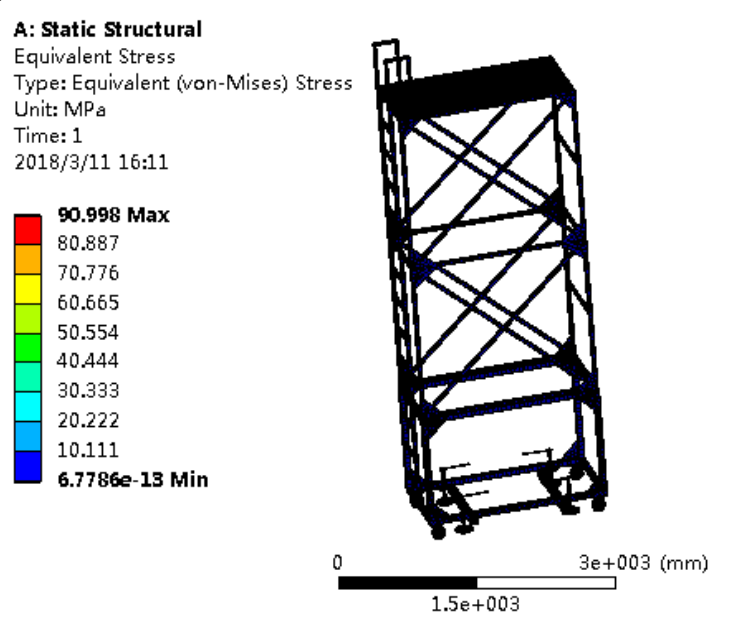

Figure 4. Stress nephogram of mobile maintenance platform.

Figure 4 shows that the maximum stress is 90.998 $\mathrm{MPa}$ and the maximum stress is less than the yield stress of the material properties, indicating that the strength of the mobile maintenance platform is sufficient. The maximum stress occurs at the chamfer of the flat plate. The main reason is that the chamfering angle is too small, therefore, the chamfering angle should increase. In order to reduce the weight of the mobile maintenance platform, the model can be lightweight and optimized design. 


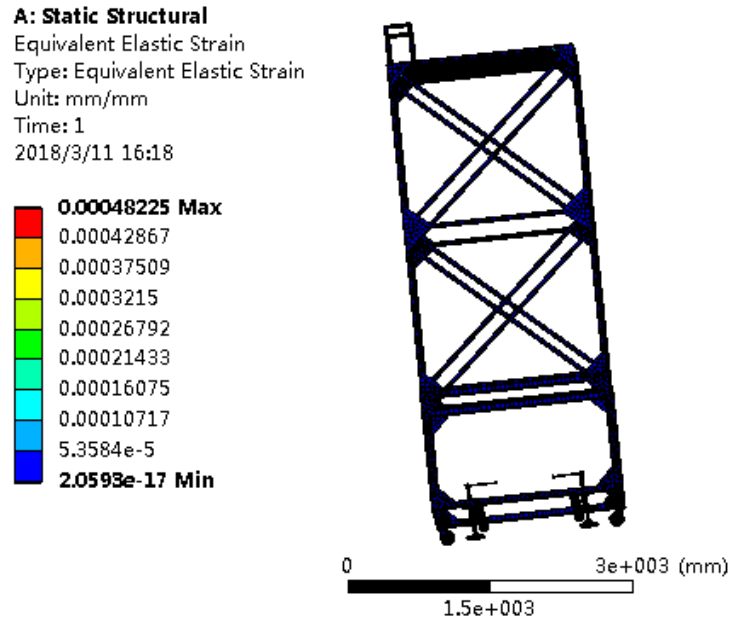

Figure 5. Strain of mobile maintenance platform.

Figure 5 shows that the maximum of the mobile platform should be 0.0005 , indicating that the material strength of the mobile maintenance platform designed in this paper is sufficient. In order to reduce the cost of the mobile maintenance platform, it is possible to select a metal material or a composite material, which is lighter and has sufficient strength for the model.

The deformation of the moving platform is shown in Figure 6.
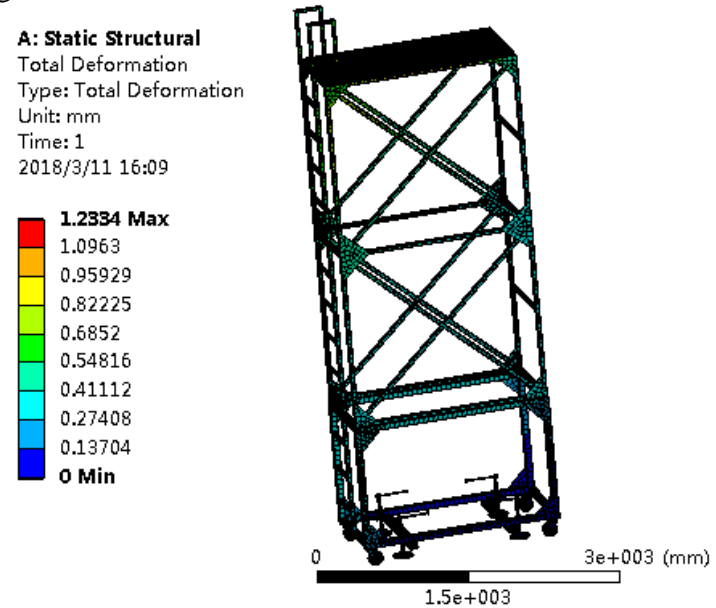

Figure 6. Deformation of mobile maintenance platform.

From Figure 6, we can see that when the mobile maintenance platform is working on three people at the same time, the maximum deformation is $1.2334 \mathrm{~mm}$, and the maximum value occurs in the center of the footrest above the mobile maintenance platform. In order to reduce the maximum deformation, we can increase the beam support at the top to reduce the amount of deformation.

\section{Fatigue analysis}

Fatigue is a material or structure under the action of alternating load, in more than one place or cause permanent damage. With the increase of cycling time, it can cause crack and fracture process ${ }^{[5-7]}$. In fatigue design, the linear fatigue damage accumulation theory is most widely used. The most typical theory is the miner damage theory ${ }^{[8]}$, which can be given by:

$$
D=\frac{n_{1} D}{N_{1}}+\frac{n_{2} D}{N_{2}}+\frac{n_{3} D}{N_{3}}
$$

where

$\mathrm{D}$ is the maximum value of the final fracture of the material;

$\mathrm{n}_{(\mathrm{i}=1,2,3)}$ is the number of cycles under the max principal stress, middle principal stress, min principal stress, respectively;

$\mathrm{N}_{(\mathrm{i}=1,2,3)}$ is the number of cycles to achieve the final fracture threshold for the max principal stress, middle principal stress, min principal stress, respectively.

The S-N curve of the material is usually made using symmetrical loads. Due to different load types, the fatigue aging is different, therefore, it needs to be corrected.

Goodman linear model:

$$
\frac{\sigma_{a}}{\sigma_{-1}}+\frac{\sigma_{m}}{\sigma_{b}}=1
$$

Gerber parabolic model:

$$
\frac{\sigma_{a}}{\sigma_{-1}}+\left(\frac{\sigma_{m}}{\sigma_{b}}\right)^{2}=1
$$

Soderberg linear model:

$$
\frac{\sigma_{a}}{\sigma_{-1}}+\frac{\sigma_{m}}{\sigma_{s}}=1
$$

The Gerber parabola model is relatively dangerous. The Goodman linear model is between the other two models. This paper selects the Goodman linear model. The relationship is shown in Figure 7.

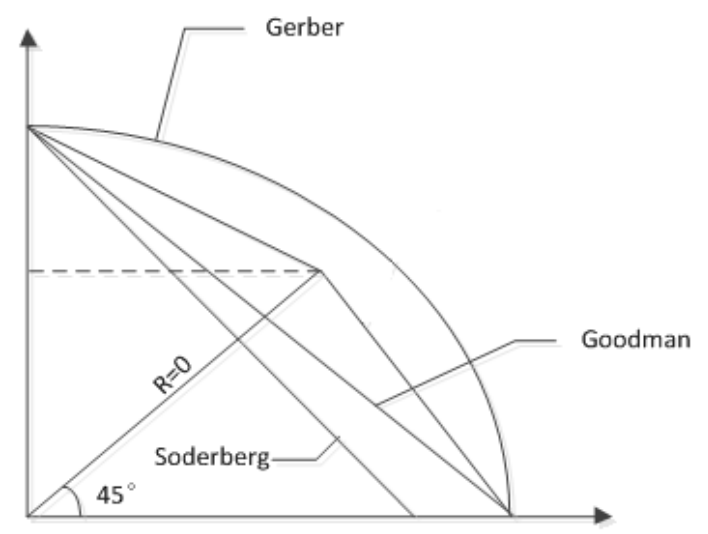

Figure 7. The Correction method of average stresses.

After simulation analysis, the life-time cloud of the mobile maintenance platform is shown in Figure 8. 


A: Static Structural
Life
Type: Life
Time: 0
$2018 / 3 / 11 \quad 16: 39$
\begin{tabular}{|l}
$2 e 7$ Max \\
$2 e 6$ \\
$2 e 5$ \\
20000 \\
2000 \\
200 \\
20 \\
2 \\
0.2 \\
0 Min
\end{tabular}

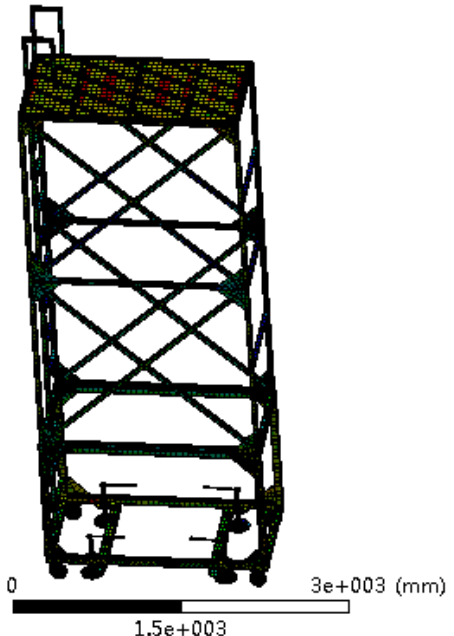

Figure 8. Life-time of the mobile maintenance platform.

From Figure 8, we can see that the maximum number of cycles for the mobile maintenance platform is $2 \mathrm{E} 7$. Assume that the mobile maintenance platform works 5 hours a day and the working time is 350 days a year. Then the fatigue life of the boom is given by:

$$
\mathrm{T}=\mathrm{N} \times \mathrm{t} /(60 \times 60 \times 8 \times 350)=19.8
$$

where

$\mathrm{N}$ is the number of cycles;

$t$ is the time of each cycle.

\section{Conclusion}

Utilizing UG to design the mobile maintenance platform and complete the establishment of the mobile maintenance platform. To obtain the deformation, stress, and strain of the mobile maintenance platform, this paper uses the Ansys/Workbench to complete the calculation. The results show that the strength of the mobile platform is sufficient. In order to reduce the weight of the mobile maintenance platform, the model can be reduced in weight.

Fatigue analysis is carried on the mobile maintenance platform using the fatigue module based on the cumulative fatigue damage theory. The fatigue life of the mobile maintenance platform has been obtained. Combined with the results of finite element analysis, the practical significance of the future lightweight design will provide the basis for future engineering design.

\section{References}

1. L.J. Zhang. J. Qinghai. Univ. National. 315 (2011).

2. L. Nie, Y.N. Zhong, Y.P. Zhang. et al. J. Hubei. Eng. Univ. 174 (2002).

3. J.W. Zhao, H.Y. Wang. Structural Finite Element Analysis. Science press, (2009).

4. T.D. Cha, P. Yang. Coal. Mine Mech. 332 (2012).

5. Q.J. Zhang. Xi`an University of Architecture and Technology, (2015).
6. D.T. Qin, L.Y. Xie. Fatigue strength and reliability design. Chemical industry press, (2013) .

7. Q. Su. Central South University, (2014).

8. Carpinteri A, Spagnoli A. Int. J. Fatigue. 315 (2009). 